Article

\title{
Oviposition Deterrent and Larvicidal and Pupaecidal Activity of Seven Essential Oils and their Major Components against Culex quinquefasciatus Say (Diptera: Culicidae): Synergism-antagonism Effects
}

\author{
Sergio Andrade-Ochoa ${ }^{1,2}$, Daniela Sánchez-Aldana ${ }^{3}$, Karla Fabiola Chacón-Vargas ${ }^{2}$, \\ Blanca E. Rivera-Chavira ${ }^{1}$, Luvia E. Sánchez-Torres ${ }^{2}$ (D), Alejandro D. Camacho ${ }^{2}$, \\ Benjamín Nogueda-Torres ${ }^{2}$ and Guadalupe Virginia Nevárez-Moorillón ${ }^{1, *}$ \\ 1 Facultad de Ciencias Químicas, Universidad Autónoma de Chihuahua, Circuito Universitario S/N, Campus \\ Universitario II. Chihuahua, Chihuahua 31170, Chihuahua, México; s.andrade.rat@gmail.com (S.A.-O.); \\ bchavira@uach.mx (B.E.R.-C.) \\ 2 Escuela Nacional de Ciencias Biológicas, Instituto Politécnico Nacional. Prolongación de Carpio y Plan de \\ Ayala S/N. Col. Santo Tomas 11340, México, DF, México; faby.chv@gmail.com (K.F.C.-V.); \\ luviasanchez@hotmail.com (L.E.S.-T.); adcamachov18@gmail.com (A.D.C.); bnogueda@gmail.com (B.N.-T.) \\ 3 Departamento de Investigación de Alimentos. Facultad de Ciencias Químicas. \\ Universidad Autónoma de Coahuila, Saltillo 25000, Coahuila, México; danielasav9@gmail.com \\ * Correspondence: vnevare@uach.mx; Tel.: +52-614-236-60-00 (ext. 4248)
}

Received: 6 January 2018; Accepted: 12 February 2018; Published: 14 February 2018

\begin{abstract}
The larvicidal activity of essential oils cinnamon (Cinnamomum verum J. Presl), Mexican lime (Citrus aurantifolia Swingle) cumin (Cuminum cyminum Linnaeus), clove (Syzygium aromaticum (L.) Merr. \& L.M.Perry), laurel (Laurus nobilis Linnaeus), Mexican oregano (Lippia berlandieri Schauer) and anise (Pimpinella anisum Linnaeus)) and their major components are tested against larvae and pupae of Culex quinquefasciatus Say. Third instar larvae and pupae are used for determination of lethality and mortality. Essential oils with more than 90\% mortality after a 30-min treatment are evaluated at different time intervals. Of the essential oils tested, anise and Mexican oregano are effective against larvae, with a median lethal concentration $\left(\mathrm{LC}_{50}\right)$ of 4.7 and $6.5 \mu \mathrm{g} / \mathrm{mL}$, respectively. Anise essential oil and $t$-anethole are effective against pupae, with $\mathrm{LC}_{50}$ values of 102 and $48.7 \mu \mathrm{g} / \mathrm{mL}$, respectively. Oregano essential oil and carvacrol also have relevant activities. A kinetic analysis of the larvicidal activity, the oviposition deterrent effect and assays of the effects of the binary mixtures of chemical components are undertaken. Results show that anethole has synergistic effects with other constituents. This same effect is observed for carvacrol and thymol. Limonene shows antagonistic effect with $\beta$-pinene. The high larvicidal and pupaecidal activities of essential oils and its components demonstrate that they can be potential substitutes for chemical compounds used in mosquitoes control programs.
\end{abstract}

Keywords: Essential oils; disease vector mosquitoes; larvicidal activity; repellency; terpenes; synergism effects; Culex quinquefasciatus

\section{Introduction}

A large proportion of human population is exposed to infectious diseases spread by mosquitoes, including Culex spp., Anopheles spp. and Aedes spp., which are vectors of parasitic diseases [1]. Culex quinquefasciatus Say is a vector of lymphatic filariasis. The disease is widely distributed in tropical regions, with around 150 million people infected, ranking filariasis as one of the main causes of global 
morbidity [2]. Culex quinquefasciatus is present in tropical areas, a household pest in many urban settings, and widely reported as a vector resistant to registered insecticides [3].

Tropical areas are more prone to vector-borne parasitic diseases and the risk has increased due to the intensification of globalization [4]. Changes in climatic conditions due to global warming have promoted favorable conditions (temperature and humidity) for the development of mosquito populations, including hematophagous species. Poor sanitary conditions for water storage intended to household use, as well as unsanitary management of urban solid waste, can increase mosquito populations in urban and rural areas [1].

On the other hand, the intensive use of synthetic insecticides in mosquito control programs has created resistance in the insect population [5], undesirable effects on other insects [6] and negative impacts on the environment [7]. Although the use of insecticide is the main method in the global effort for mosquito control [8], vector-borne diseases are persistent due to mosquitoes resistant to traditionally used insecticides [9] and lack of effective vaccines [10]. The resistance of mosquitoes to synthetic insecticides has led to an interest in natural products with potential insecticidal activity, especially those derived from plants, which are considered valid alternatives to conventional synthetic pesticides to control a variety of insect pests and vectors. In this context, the essential oils and their constituents have received much attention as potentially useful bioactive compounds against insects [11]. The complex and variable mixtures of bioactive compounds with different modes of action, offered by plants, may lessen the chance of resistance in mosquito populations [12].

Essential oils are heterogeneous mixtures of organic chemical compounds from different chemical families [13]; many have a terpenoid backbone, especially monoterpenes and sesquiterpenes. Low molecular weight aliphatic compounds, acyclic esters or lactones may also be present [14]. Chemical composition of essential oils is affected by factors such as plant species and subspecies, geographical location, harvest time, part of the plant used and the extraction methods used to obtain the essential oil [15]. Several studies have reported the larvicidal action of medicinal plants and their essential oils against insect vectors of Culex and Anopheles genera, as well as Aedes aegypti (L.) [16,17]. Furthermore, there are many secondary metabolites found in most of those plants that are considered to have insecticidal properties $[18,19]$.

The aim of this paper is to determine, by laboratory bioassays, the larvicidal activity of seven essential oils on third instar larva and pupa of C. quinquefasciatus. Essential oils tested include cinnamon (Cinnamomum verum J. Presl), Mexican lime (Citrus aurantifolia Swingle) cumin (Cuminum cyminum Linnaeus), clove (Syzygium aromaticum (L.) Merr. \& L.M. Perry), laurel (Laurus nobilis Linnaeus), Mexican oregano (Lippia berlandieri Schauer) and anise (Pimpinella anisum Linnaeus). Larvicidal effect and synergistic behavior of the major components present in the essential oils included in the study are also tested.

\section{Materials and Methods}

\subsection{Insect Cultures and Rearing Conditions}

Larvae of C. quinquefasciatus were collected from water tanks in the Sanctorum Cemetery in Mexico City, Mexico $\left(19^{\circ} 27^{\prime} 17^{\prime \prime} \mathrm{N}, 99^{\circ} 12^{\prime} 47^{\prime \prime} \mathrm{W}\right)$. Identification of adults and larvae was done based on Harwood and James descriptions [20]. Mosquitoes larvae were pooled into groups of 50 individuals of first and second instars in glass bottles containing distilled water. Afterwards, larvae were maintained at $26 \pm 2{ }^{\circ} \mathrm{C}$ with a natural photoperiod and supplied with powdered mixture of dog food and baking powder (3:1). The third instar emerging larvae were then separated into groups of 10 individuals in $100 \mathrm{~mL}$ tubes containing distilled water.

\subsection{Plant material}

For the vegetative materials used in this study, cinnamon cortex (Cinnamomum verum), cumin leaves (Cuminum cyminum), clove bottoms (Syzygium aromaticum), laurel leaves (Laurus nobilis), 
Mexican oregano leaves (Lippia berlandieri) and anise seeds (Pimpinella anisum) were purchased as commercial spices from Commercial Cardona S. A. (Chihuahua Chihuahua, México). Mexican lime (Citrus aurantifolia) bagasse was obtained from fruits brought at a local supermarket. Pure chemical compounds identified as major components of the essential oils were purchased from a Sigma-Aldrich (San Louis, MI, USA) distributor.

\subsection{Essential Oils Extraction and Characterization}

A modified Schilcher apparatus was used for hydrodistillation; $200 \mathrm{~g}$ of plant material (dried and chopped) was added to $4 \mathrm{~L}$ of water and introduced into the boiling flask and the system was heated at $100{ }^{\circ} \mathrm{C}$ for $5 \mathrm{~h}$ [21,22]; for Mexican lime essential oil, $400 \mathrm{~g}$ of fresh lime bagasse and $4 \mathrm{~L}$ of water were used and the system was heated at $100{ }^{\circ} \mathrm{C}$ for $4 \mathrm{~h}$ (Aldana et al. 2014 [23]); the essential oil extracted was dried over anhydrous sodium sulfate and stored at $4{ }^{\circ} \mathrm{C}$ in amber glass vials. For chemical analysis, conditions were similar to those reported by Aldana et al. [23] for gas chromatographic separation followed by mass spectrometry. Analysis were done in a Perkin Elmer AUTOSYSTEM XL Gas Chromatograph (Waltham, MA, USA) and TurboMass Gold Spectrometer (Hewlett-Packard In., Palo Alto, CA, USA).

\subsection{Bioassays and Statistical Analysis}

Mosquito larvicidal assays were carried out according to standard World Health Organization (WHO) larvicidal assay method with slight modifications [24]. The essential oils and the binary mixtures of the most effective essential oils (i.e., the oils which achieved the lowest $\mathrm{LC}_{50}$ values on C. quinquefasciatus larvae) were diluted in dimethyl sulfoxide (DMSO) (Sigma-Aldrich, San Louis, MI, USA) preparing a serial dilution of test dosages. For each experimental treatment, $1 \mathrm{~mL}$ of serial dilution was added to $224 \mathrm{~mL}$ of distilled water in a 500-mL glass bowl and shaken lightly to ensure a homogenous test solution. The selected larvae were transferred in distilled water into a bowl of prepared test solution with final surface area of $125 \mathrm{~cm}^{2}$ (25 larvae/beaker) and maintained in starvation throughout the experimental period; the surviving larvae were counted to record larval mortality. Five replicates were run simultaneously with at least 10 dosages (300, 200, 100, 75, 50, $25,15,10,5$, and $2.5 \mu \mathrm{g} / \mathrm{mL}$ ). The larvicidal activity of DMSO was also determined under the same conditions; a DMSO concentration of $1000 \mu \mathrm{g} / \mathrm{mL}$ had no larvicidal activity. The lethal concentrations (LC 50 and $\mathrm{LC}_{90}$ ) were calculated using Probit analysis. Data were processed using MS Excel 2010 (Microsoft, WA, USA) and SAS v.9 (Proc Probit) (SAS Institute Inc. Cary, NC, USA) computer programs.

\subsection{The Effect of Lethal Doses on Larval Development}

Twenty-five larvae (third instar) were put into a 500-mL glass bowl containing $200 \mathrm{~mL}$ drinking water. Upon acclimatization (after approximately $1 \mathrm{~h}$ ), a dose of essential oil or pure components was mixed to the water, corresponding to the calculated dose $\mathrm{LC}_{50}$. The essential oils and pure compounds were emulsified using DMSO; water with an adequate DMSO content was used for the control larvae. Larval mortality was observed $24 \mathrm{~h}$ following termination of exposure, during which time, no food was offered to the larvae. Five replicates were run simultaneously.

\subsection{Oviposition Deterrent Effect}

The oviposition deterrent test was done using the method described by Xue et al. [25]. The percent of effective repellency for was calculated using the formula:

$$
E R(\%)=((N C-N T) /(N C+N T) \times 100
$$

where $E R$ is the percent of effective repellency; $N C$ is the number of eggs in the control sample; and NT is the number of eggs in treatment bowl. ER was determined and transformed to arcsine square root values for analysis of variance. 


\subsection{Effects of Dose and Time Period on Larvicidal Activity}

Essential oils that yielded more than $90 \%$ mortality after $30 \mathrm{~min}$ of treatment in preliminary screening were further evaluated at different concentrations from 2.5 to $200 \mu \mathrm{g} / \mathrm{mL}$ at different time intervals (10 to $120 \mathrm{~min}$ ) for the determination of $\mathrm{LC}_{50}$ and $\mathrm{LC}_{90}$ values. Larvae were considered dead if they were immobile and unable to reach the water surface [26].

\subsection{Effects of the Binary Mixtures}

Three test groups were run concurrently for each binary combination tested: the binary mixture and each of the pure compounds. The compounds were combined in a 1:1 ratio (doses $\mathrm{LC}_{25} / \mathrm{LC}_{25}$ ). The application method and experimental conditions were identical to the methods described previously in Section 2.4. Four replications of 25 larvae were tested per dose.

Actual mortalities were compared to expected mortalities based on the formula:

$$
E=O_{a}+O_{b}\left(1-O_{a} / 100\right)
$$

where $E$ is the expected mortality and $O_{a}$ and $O_{b}$ are the observed mortalities of pure compounds at the given concentration. The factor of 100 was used to calculate the value of $E$.

The effects of mixtures were designated as either antagonistic, additive, or synergistic by analysis using $x^{2}$ comparisons:

$$
x^{2}=\left(O_{m}-E\right)^{2} / E
$$

where $\mathrm{O}_{\mathrm{m}}$ is the observed mortality from the binary mixture and $\mathrm{E}$ is the expected mortality, $x^{2}$ with $d f=1$, and $p=0.05$ is 3.84. A pair with $x^{2}$ values $>3.84$ and having greater than expected mortality were considered to be synergistic (or antagonistic), with $x^{2}$ values $<3.84$ representing additive effects $[19,27]$.

\section{Results}

\subsection{Essential Oils Extraction and Characterization}

Characterization of the essential oils has been reported previously by our research group [21-23]. Major components of each essential oil are included in Table 1 and their structures are shown in Figure 1.

Table 1. Main essential oil components of the seven essential oils investigated for larvicidal toxicity against the filariasis vector Culex quinquefasciatus.

\begin{tabular}{cc}
\hline Essential Oil & Major components \\
\hline Cinnamomum verum J. Presl & Cinnamaldehyde $(98.3 \%), 3$-carene $(0.3 \%), \beta$-caryophyllene $(0.1 \%)$ \\
Citrus aurantifolia Swingle & Limonene $(98.6 \%), \beta$-pinene $(0.1 \%), \alpha$-pinene $(0.1 \%)$ \\
Cuminum cyminum Linnaeus & Cuminaldehyde $(89.6 \%), \alpha$-pinene $(3.2 \%)$, limonene $(1.1 \%)$ \\
Syzygium aromaticum (L.) Merr. \& L.M. Perry & Eugenol $(96.3 \%)$, chavicol $(1.23 \%), \beta$-caryophyllene $(1.1 \%)$ \\
Laurus nobilis Linnaeus & Eucalyptol $(76.1 \%), \alpha$-pinene $(5.7 \%) \beta$-myrcene $(4.6 \%)$ \\
Lippia berlandieri Shauer & Carvacrol $(57.5 \%)$, thymol $(32.8 \%), p$-cymene $(1.8 \%)$ \\
Pimpinella anisum Linnaeus & t-Anethole $(78.0 \%), \beta$-myrcene $(15.3 \%)$, limonene $(2.1 \%)$ \\
\hline
\end{tabular}


1)<smiles>C/C=C/c1ccc(OC)cc1</smiles><smiles>C=C/C=C/c1ccccc1</smiles>

3)<smiles>Cc1ccc(C(C)C)cc1O</smiles>

4)<smiles>CC(C)c1ccc(C=O)cc1</smiles>

10)
5)<smiles>Cc1ccc(C(C)C)cc1</smiles>

6)<smiles>CC(C)C1CCC2(C)CCC1O2</smiles>

7)<smiles>C=CCc1ccc(O)c(OC)c1</smiles>

8)<smiles>C=C(C)C1CC=C(C)CC1</smiles>

9)<smiles>C=CC(=C)CCC=C(C)C</smiles>

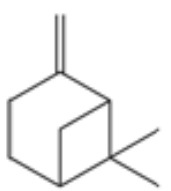

11)<smiles>Cc1ccc(C(C)C)c(O)c1</smiles>

Figure 1. Chemical structure of major components (these are analytical standards purchased from Sigma-Aldrich as mentioned in the experimental section) evaluated: (1) trans-Anethole; (2) Cinnamaldehyde; (3) Carvacrol; (4) Cuminaldehyde; (5) p-Cymene; (6) Eucalyptol; (7) Eugenol; (8) (-)-Limonene; (9) $\beta$-Myrcene;

(10) $\beta$-Pinene; and (11) Thymol.

\subsection{Larvicidal and Pupaecidal Activities of Essential Oils and Their Constituents}

The results of in vitro assays demonstrated the larvicidal activity of essential oils against C. quinquefasciatus (Diptera: Culicidae), since all oils tested were able to eliminated $100 \%$ of larvae at $75 \mu \mathrm{g} / \mathrm{mL}$. The essential oils of Mexican oregano and anise display the highest larvicidal activity with $\mathrm{LC}_{50}$ of 6.21 and $4.62 \mu \mathrm{g} / \mathrm{mL}$, respectively. Pupae are more resistant than larvae, since more than $100 \mu \mathrm{g} / \mathrm{mL}$ of essential oil were needed to reach $\mathrm{LC}_{50}$; the same effect has been reported before [28,29]. Tables 2 and 3 show lethal concentrations $\mathrm{LC}_{50}$ and $\mathrm{LC}_{90}$ of the essential oils and their constituents studied against larval stage III and pupae of $C$. quinquefasciatus after $24 \mathrm{~h}$ exposure.

As observed, $t$-Anethole, which is the main compound of anise essential oil, proved to be efficient against larvae as well as pupae, with $\mathrm{LC}_{50}$ of 7.4 and $28.6 \mu \mathrm{g} / \mathrm{mL}$, respectively. Carvacrol also showed efficient activity against larvae and pupae, while thymol showed lower activity, with an $\mathrm{LC}_{50}$ of $23.4 \mu \mathrm{g} / \mathrm{mL}$ against larvae and $100.5 \mu \mathrm{g} / \mathrm{mL}$ against pupae. Eugenol and eucalyptol show larvicidal activity with a $\mathrm{LC}_{50}$ of 23.04 and $24.83 \mu \mathrm{g} / \mathrm{mL}$, respectively. None of the aforementioned compounds had significant activity against pupae. Percentage of Culex quinquefasciatus larvae mortality upon exposure to water contaminated by $\mathrm{LC}_{50}$ doses is shown in Table 4. 
Table 2. $\mathrm{LC}_{50}$ and $\mathrm{LC}_{90}(\mu \mathrm{g} / \mathrm{mL})$ of essential oils on Culex quinquefasciatus at III instar and pupal stages after $24 \mathrm{~h}$ of exposure.

\begin{tabular}{|c|c|c|c|c|c|c|}
\hline \multirow{2}{*}{ Essential Oils } & \multicolumn{3}{|c|}{ III Instar } & \multicolumn{3}{|c|}{ Pupae } \\
\hline & $\mathrm{LC}_{50}$ & $\mathrm{LC}_{90}$ & Chi ${ }^{* *}$ & $\mathrm{LC}_{50}$ & $\mathrm{LC}_{90}$ & Chi ** \\
\hline Cinnamomum verum J. Presl & $24.5(23.2-25.8)$ & $53.7(52.2-55.1)$ & 1.198 & $216.7(212.8-220.6)$ & $374.1(359.8-388.3)$ & 0.055 \\
\hline Citrus aurantifolia Swingle & $14.1(13.9-14.3)$ & $30.9(28.4-33.4)$ & 0.039 & $184.9(180.0-189.7)$ & $381.0(365.6-396.5)$ & 1.331 \\
\hline Cuminum cyminum Linnaeus & $23.3(21.8-24.9)$ & $56.3(52.4-60.1)$ & 0.048 & $98.4(95.9-101.3)$ & $212.6(199.5-225.8)$ & 0.020 \\
\hline $\begin{array}{l}\text { Syzygium aromaticum (L.) } \\
\text { Merr. \& L.M. Perry }\end{array}$ & $22.5(21.5-23.7)$ & $49.7(47.0-52.4)$ & 1.326 & $236.5(232.5-240.4)$ & $447.0(433.7-460.3)$ & 1.202 \\
\hline Laurus nobilis Linnaeus & $49.0(48.5-50.5)$ & $111.0(107.0-115.0)$ & 0.674 & $204.9(201.8-208.1)$ & $460.1(442.4-447.8)$ & 0.571 \\
\hline Lippia berlandieri Shauer & $6.5(5.9-6.9)$ & $18.8(17.8-19.8)$ & 0.268 & $181.0(178.4-183.6)$ & $356.1(341.0-371.1)$ & 0.645 \\
\hline Pimpinella anisum Linnaeus & $4.6(3.5-5.8)$ & $9.0(8.5-9.5)$ & 2.572 & $51.6(49.2-54.0)$ & $102.0(85.1-118.8)$ & 0.016 \\
\hline Temephos H (control) & $2.1(1.8-2.5)$ & $5.2(4.1-6.4)$ & 0.039 & $34.0(29.1-39.0)$ & $49.2(43.4-54.9)$ & 0.048 \\
\hline
\end{tabular}

In parenthesis, $95 \%$ confidence intervals, essential oils activity is considered significantly different when the $95 \%$ CI fail to overlap. ${ }^{* *}$ Chi-square value, significant at $p<0.05$ level.

Table 3. $\mathrm{LC}_{50}$ and $\mathrm{LC}_{90}(\mu \mathrm{g} / \mathrm{mL})$ of the major constituents of the essential oils at III instar and pupal of Culex quinquefasciatus after $24 \mathrm{~h}$ of exposure.

\begin{tabular}{ccccccc}
\hline \multirow{2}{*}{ Compounds } & \multicolumn{3}{c}{ III Instar } & \multicolumn{3}{c}{ Pupae } \\
\cline { 2 - 7 } & LC $_{\mathbf{5 0}}$ & LC $_{\mathbf{9 0}}$ & Chi $^{* *}$ & LC $_{\mathbf{5 0}}$ & LC $_{\mathbf{9 0}}$ & Chi $^{* *}$ \\
\hline$t$-anethole & $7.4(6.5-8.3)$ & $18.8(17.4-20.2)$ & 0.055 & $28.6(26.4-30.8)$ & $48.6(45.79-51.51)$ & 0.039 \\
carvacrol & $5.5(5.2-5.7)$ & $11.3(9.9-2.7)$ & 2.684 & $53.2(49.9-56.6)$ & $111.4(102.0-120.8)$ & 0.483 \\
cinnamaldehyde & $18.4(17.6-19.2)$ & $39.2(35.2-43.1)$ & 1.203 & $90.1(84.6-95.6)$ & $179.3(170.4-188.1)$ & 0.048 \\
cuminaldehyde & $23.0(21.9-24.1)$ & $52.2(50.1-54.3)$ & 4.313 & $95.4(91.1-99.6)$ & $198.2(193.7-202.7)$ & 0.896 \\
$p$-cymene & $23.1(21.2-24.9)$ & $91.9(89.7-94.0)$ & 0.253 & $306.3(297.4-315.1$ & $549.2(533.0-565.3)$ & 1.326 \\
eucalyptol & $24.8(22.7-26.8)$ & $48.0(44.9-51.1)$ & 0.116 & $92.9(89.1-96.6)$ & $193.9(186.9-201.0)$ & 0.574 \\
eugenol & $23.0(21.7-24.3)$ & $51.2(46.6-55.8)$ & 0.279 & $106.9(99.5-114.3)$ & $198.4(189.6-207.1)$ & 0.665 \\
(-)-limonene & $14.2(13.5-15.0)$ & $36.4(33.5-39.4)$ & 0.572 & $78.4(73.3-83.4)$ & $155.0(145.6-164.3)$ & 0.865 \\
myrcene & $19.5(18.5-20.4)$ & $40.0(36.7-43.2)$ & 2.561 & $31.8(30.4-33.1)$ & $59.1(55.2-62.9)$ & 1.287 \\
$\beta$-pinene & $19.6(18.8-20.3)$ & $61.9(57.8-65.9)$ & 0.265 & $206.9(95.3-105.8)$ & $458.4(440.1-476.6)$ & 3.404 \\
thymol & $23.4(22.5-24.4)$ & $45.4(43.3-47.6)$ & 0.683 & $100.57(88.3-111.8)$ & $168.7(155.8-181.6)$ & 1.642 \\
\hline
\end{tabular}

In parenthesis, $95 \%$ confidence intervals, essential oils activity is considered significantly different when the $95 \%$ CI fail to overlap. ${ }^{* *}$ Chi-square value, significant at $p<0.05$ level. 
Table 4. Percentage of Culex quinquefasciatus larvae mortality upon exposure to water contaminated by $\mathrm{LC}_{50}$ doses.

\begin{tabular}{|c|c|c|c|}
\hline Essential Oils & Larval Mortality & Compounds & Larval Mortality \\
\hline Cinnamomum verum J. Presl & $52.7 \pm 7.6^{\mathrm{a}}$ & cinnamaldehyde & $62.3 \pm 9.1^{\mathrm{a}}$ \\
\hline Citrus aurantifolia Swingle & $54.7 \pm 5.8^{\mathrm{abc}}$ & $\begin{array}{c}\text { (-)-limonene } \\
\beta \text {-pinene }\end{array}$ & $\begin{array}{l}56.2 \pm 9.7^{\mathrm{ab}} \\
49.6 \pm 7.3^{\mathrm{ab}}\end{array}$ \\
\hline Cuminum cyminum Linnaeus & $52.2 \pm 0.7^{\mathrm{abc}}$ & cuminaldehyde & $56.2 \pm 11.7^{\mathrm{ab}}$ \\
\hline Syzygium aromaticum (L.) Merr. \& L.M. Perry & $52.9 \pm 4.9 \mathrm{abc}$ & eugenol & $56.7 \pm 7.4^{\mathrm{ab}}$ \\
\hline Laurus nobilis Linnaeus & $65.3 \pm 10.9^{\mathrm{a}}$ & eucalyptol & $63.8 \pm 4.8^{\mathrm{a}}$ \\
\hline Lippia berlandieri Shauer & $60.7 \pm 5.8^{a b}$ & $\begin{array}{c}\text { carvacrol } \\
\text { thymol }\end{array}$ & $\begin{array}{l}58.7 \pm 6.6^{\mathrm{ab}} \\
48.7 \pm 3.4^{\mathrm{ab}}\end{array}$ \\
\hline Pimpinella anisum Linnaeus & $48.2 \pm 6.1^{b c}$ & $\begin{array}{l}t \text {-anethole } \\
\text { myrcene }\end{array}$ & $\begin{array}{l}47.1 \pm 5.8^{a b} \\
58.7 \pm 8.3^{a b}\end{array}$ \\
\hline Temephos H (control) & $46.8 \pm 6.5^{\mathrm{c}}$ & Temephos H (control) & $43.3 \pm 7.6^{b}$ \\
\hline Control (Water) & $0.0 \pm 0.0$ & Control (Water) & $0.0 \pm 0.0$ \\
\hline
\end{tabular}

Numbers in column followed by different letters are significantly different at level of $p<0.05$ according to Tukey's test.

Table 5. Oviposition deterrent activity of P. anisum, L. berlandieri and C.aurantifolia essential oils and their major components against gravid female Culex quinquefasciatus.

\begin{tabular}{cccccccc}
\hline \multicolumn{7}{c}{ Effective repellency (\%) } \\
\hline $\begin{array}{c}\text { Concentration } \\
\mathbf{( \% )}\end{array}$ & $\begin{array}{c}\text { P. anisum } \\
\text { Essential Oil }\end{array}$ & Anethole & $\begin{array}{c}\text { L. berlandieri } \\
\text { Essential Oil }\end{array}$ & Thymol & Carvacrol & $\begin{array}{c}\text { C. aurantifolia } \\
\text { Essential Oil }\end{array}$ & Limonene \\
\hline 0.02 & $100 \pm 0.0^{\mathrm{a}}$ & $100 \pm 0.0^{\mathrm{a}}$ & $100 \pm 0.0^{\mathrm{a}}$ & $100 \pm 0.0^{\mathrm{a}}$ & $100 \pm 0.0^{\mathrm{a}}$ & $100 \pm 0.0^{\mathrm{a}}$ & $100 \pm 0.0^{\mathrm{a}}$ \\
0.01 & $100 \pm 0.0^{\mathrm{a}}$ & $95.2 \pm 4.5^{\mathrm{b}}$ & $100 \pm 0.0^{\mathrm{a}}$ & $87.5 \pm 9.5^{\mathrm{b}}$ & $100 \pm 0.0^{\mathrm{a}}$ & $99.3^{\mathrm{a}} \pm 0.9^{\mathrm{a}}$ & $89.1 \pm 8.5^{\mathrm{b}}$ \\
0.005 & $68.6 \pm 4.4^{\mathrm{b}}$ & $49.0 \pm 5.2^{\mathrm{c}}$ & $38.0 \pm 6.3^{\mathrm{b}}$ & $18.9 \pm 6.3^{\mathrm{c}}$ & $41.3 \pm 2.5^{\mathrm{b}}$ & $23.6^{\mathrm{b}} \pm 1.8^{\mathrm{b}}$ & $17.6 \pm 3.3^{\mathrm{c}}$ \\
\hline
\end{tabular}

Each value represents the mean ( $\pm S E$ ) of five values. Values with different letters are significantly different at $p<0.05$ level (Tukey's test of multiple comparison). 


\subsection{Oviposition Deterrent Activities of Essential Oils and Their Constituents}

Oviposition deterrent activity was evaluated for the essential oils of anise, oregano and lemon, as well as its constituents, since these three essential oils were the ones with more relevant lethal potential against $C$. quinquefasciatus. As shown in Table 5, an almost $100 \%$ deterrence of female oviposition was observed for all essential oils tested in concentrations of $0.02 \%$ and $0.01 \%$. Noteworthy differences were seen only with a concentration of $0.005 \%$, where anise essential oil was again most efficient (repellency about $68 \%$ ). Results demonstrate the high potential of these compounds to control mosquitoes; therefore, these essential oils can be considered as promising agents for the development of botanical larvicides.

\subsection{Effect of Dose and Time on Larvicidal Activity}

To demonstrate the effect of essential oils and their constituents on short-term exposures, dose-response kinetics was undertaken. In the present study, cases of instantaneous death were observed within $20 \mathrm{~min}$ of exposure. The essential oil of anise can eliminate $100 \%$ of larvae in $120 \mathrm{~min}$ at a concentration of $15 \mu \mathrm{g} / \mathrm{mL}$, while $\mathrm{LC}_{50}$ is $5 \mu \mathrm{g} / \mathrm{mL}$; on the other hand, trans-anethole, which is the major component of anise essential oil, does not remove $100 \%$ of the larvae even at concentrations higher than its $\mathrm{LC}_{50}$ (Figure 2). Similarly, the Mexican oregano essential oil can eliminate $100 \%$ of the larvae in $20 \mathrm{~min}$ at a concentration of $15 \mu \mathrm{g} / \mathrm{mL}$. Similar results were obtained with carvacrol, one of its major components; however, thymol, another essential oil constituent, only eliminates $36 \%$ of larvae in $120 \mathrm{~min}$ (Figure 3).
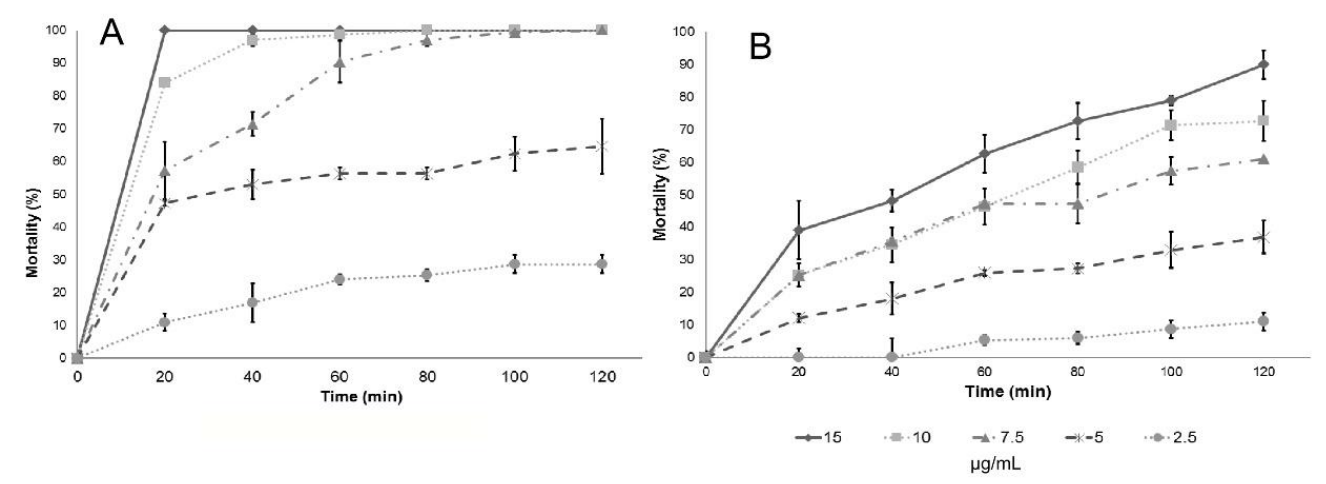

Figure 2. Effect of dose and incubation time on larval mortality of: (A) anise (Pimpinella anisum Linneaus) essential oil; and (B) trans-Anethole.

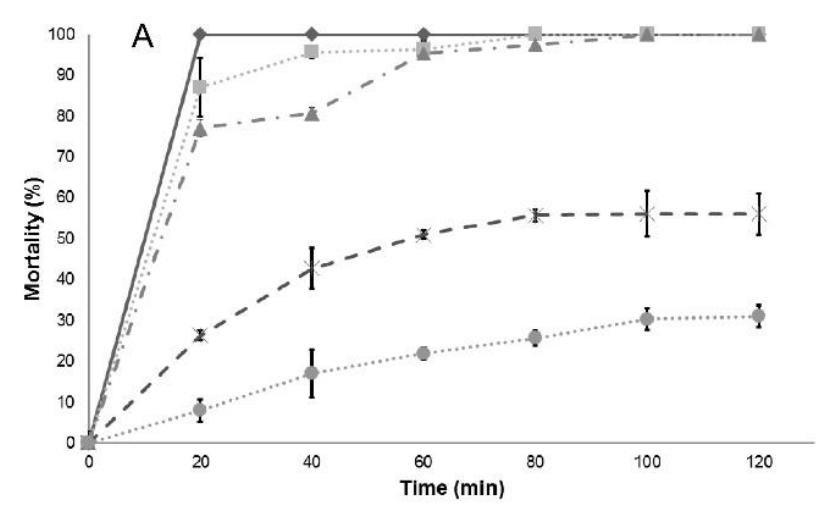

Figure 3. Cont. 

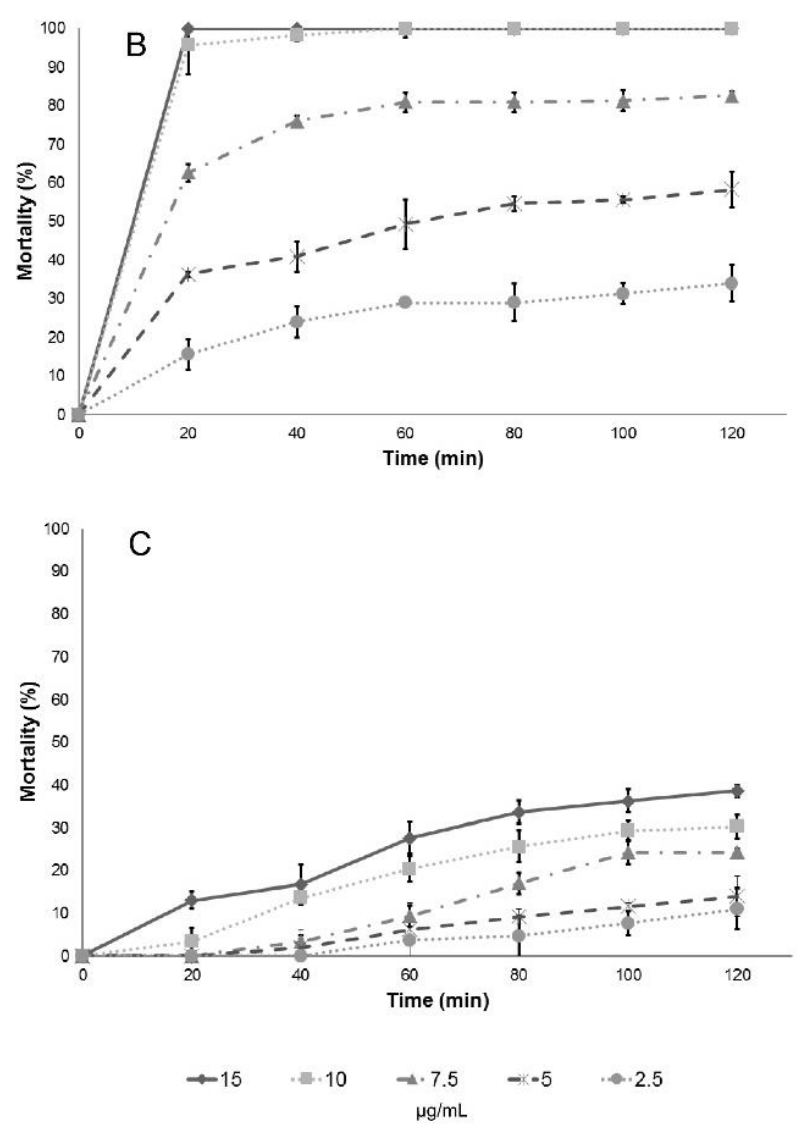

Figure 3. Effect of dose and incubation time on larval mortality of: L. berlandieri Schauer essential oils (A); and its major constituents: carvacrol (B); and thymol (C).

\subsection{Synergism-Antagonism Effect}

The difference between the activities of the essential oils and the pure constituents, as well as the results of lethality kinetics, were the basis for the determination of synergistic/antagonistic effects among the compounds tested. If synergistic effects are found, the combination of the compounds involved can provide effective biological activity at lower concentrations [19]. In the present report, 26 binary combinations were tested against larvae (III instar) (Table 6) and pupae (Table 7), of which 20 presented a significant synergistic effect $(p<0.5)$, while five showed no effect on mortality and one presented an antagonistic effect. Trans-anethole displays synergistic effects in all combinations evaluated except in combination with $\beta$-pinene (Table 6), while in the combination with thymol it did not present effects against the pupae (Table 7). 
Table 6. Effect of binary mixtures of individual compounds on the mortality against Culex quinquefasciatus larvae.

\begin{tabular}{|c|c|c|c|c|c|c|c|}
\hline \multirow{2}{*}{\multicolumn{2}{|c|}{ Compounds }} & \multicolumn{4}{|c|}{ Larval Mortality (\%) } & \multirow{3}{*}{$x^{2}$} & \multirow{3}{*}{ Effect } \\
\hline & & \multicolumn{2}{|c|}{ Pure Compounds } & \multicolumn{2}{|c|}{ Binary Mixtures } & & \\
\hline Compound A & Compound B & Observed A & Observed B & Expected & Observed & & \\
\hline$t$-anethole & carvacrol & 13.2 & 27.8 & 35.6 & 86.4 & 72.5 & Synergistic \\
\hline$t$-anethole & cinnamaldehyde & 13.2 & 8.3 & 18.7 & 71.1 & 147.3 & Synergistic \\
\hline$t$-anethole & $p$-cymene & 13.2 & 3.2 & 14.2 & 87.6 & 378.1 & Synergistic \\
\hline$t$-anethole & eugenol & 13.2 & 8.1 & 18.5 & 56.5 & 78.2 & Synergistic \\
\hline$t$-anethole & (-)-limonene & 13.2 & 6.2 & 16.8 & 98.3 & 394.1 & Synergistic \\
\hline$t$-anethole & myrcene & 13.2 & 3.3 & 14.3 & 62.7 & 163.4 & Synergistic \\
\hline$t$-anethole & $\beta$-pinene & 13.2 & 5.3 & 16.1 & 15.4 & 0.0 & No effect \\
\hline$t$-anethole & thymol & 13.2 & 12.6 & 22.4 & 74.7 & 122.2 & Synergistic \\
\hline (-)-limonene & carvacrol & 6.2 & 27.8 & 31.9 & 57.4 & 20.4 & Synergistic \\
\hline (-)-limonene & cinnamaldehyde & 6.2 & 8.3 & 13.6 & 42.7 & 62.3 & Synergistic \\
\hline (-)-limonene & $p$-cymene & 6.2 & 3.2 & 8.8 & 41.2 & 118.9 & Synergistic \\
\hline (-)-limonene & eugenol & 6.2 & 8.1 & 13.4 & 49.5 & 97.1 & Synergistic \\
\hline (-)-limonene & myrcene & 6.2 & 3.3 & 8.9 & 31.8 & 58.8 & Synergistic \\
\hline (-)-limonene & $\beta$-pinene & 6.2 & 5.3 & 10.8 & 2.1 & 7.0 & Antagonistic \\
\hline (-)-limonene & thymol & 6.2 & 12.6 & 17.6 & 16.3 & 0.1 & No effect \\
\hline carvacrol & cinnamaldehyde & 27.8 & 8.3 & 26.1 & 38.1 & 5.6 & Synergistic \\
\hline carvacrol & p-cymene & 27.8 & 3.2 & 22.4 & 28.5 & 1.7 & No effect \\
\hline carvacrol & eugenol & 27.8 & 8.1 & 25.9 & 81.3 & 118.3 & Synergistic \\
\hline carvacrol & myrcene & 27.8 & 3.3 & 22.5 & 85.5 & 177.0 & Synergistic \\
\hline carvacrol & $\beta$-pinene & 27.8 & 5.3 & 23.9 & 61.2 & 58.2 & Synergistic \\
\hline carvacrol & thymol & 27.8 & 8.3 & 26.1 & 72.6 & 83.1 & Synergistic \\
\hline thymol & cinnamaldehyde & 12.6 & 8.3 & 18.3 & 49.7 & 54.1 & Synergistic \\
\hline thymol & p-cymene & 12.6 & 3.2 & 13.8 & 14.4 & 0.0 & No effect \\
\hline thymol & eugenol & 12.6 & 8.1 & 18.1 & 67.8 & 136.6 & Synergistic \\
\hline thymol & myrcene & 12.6 & 3.3 & 13.9 & 81.6 & 329.8 & Synergistic \\
\hline thymol & $\beta$-pinene & 12.6 & 5.3 & 15.6 & 15.1 & 0.0 & No effect \\
\hline
\end{tabular}


Table 7. Effect of binary mixtures of individual compounds on the mortality against Culex quinquefasciatus pupae.

\begin{tabular}{|c|c|c|c|c|c|c|c|}
\hline \multirow{2}{*}{\multicolumn{2}{|c|}{ Compounds }} & \multicolumn{4}{|c|}{ Pupae Mortality (\%) } & \multirow{3}{*}{$x^{2}$} & \multirow{3}{*}{ Effect } \\
\hline & & \multicolumn{2}{|c|}{ Pure Compounds } & \multicolumn{2}{|c|}{ Binary Mixtures } & & \\
\hline Compound A & Compound B & Observed A & Observed B & Expected & Observed & & \\
\hline$t$-anethole & carvacrol & 20.3 & 15.7 & 28.7 & 68.4 & 55.0 & Synergistic \\
\hline$t$-anethole & cinnamaldehyde & 20.3 & 8.3 & 22.8 & 60.2 & 61.4 & Synergistic \\
\hline$t$-anethole & $p$-cymene & 20.3 & 2.5 & 18.2 & 66.4 & 128.0 & Synergistic \\
\hline$t$-anethole & eugenol & 20.3 & 2.8 & 18.4 & 40.3 & 26.0 & Synergistic \\
\hline$t$-anethole & (-)-limonene & 20.3 & 11.2 & 25.1 & 68.6 & 75.4 & Synergistic \\
\hline$t$-anethole & myrcene & 20.3 & 18.6 & 31.0 & 80.5 & 79.0 & Synergistic \\
\hline$t$-anethole & $\beta$-pinene & 20.3 & 2.7 & 18.3 & 21.2 & 0.4 & No effect \\
\hline$t$-anethole & thymol & 20.3 & 3.1 & 18.6 & 22.3 & 0.7 & No effect \\
\hline (-)-limonene & carvacrol & 11.2 & 15.7 & 23.9 & 42.3 & 14.2 & Synergistic \\
\hline (-)-limonene & cinnamaldehyde & 11.2 & 8.3 & 17.3 & 37.5 & 23.5 & Synergistic \\
\hline (-)-limonene & $p$-cymene & 11.2 & 2.5 & 12.2 & 49.3 & 113.3 & Synergistic \\
\hline (-)-limonene & eugenol & 11.2 & 2.8 & 12.4 & 36.4 & 46.2 & Synergistic \\
\hline (-)-limonene & myrcene & 11.2 & 18.6 & 26.5 & 61.3 & 45.9 & Synergistic \\
\hline (-)-limonene & $\beta$-pinene & 11.2 & 2.7 & 12.3 & 41.3 & 67.9 & Antagonistic \\
\hline (-)-limonene & thymol & 11.2 & 3.1 & 12.7 & 11.3 & 0.2 & No effect \\
\hline carvacrol & cinnamaldehyde & 15.7 & 8.3 & 20.2 & 21.3 & 0.1 & No effect \\
\hline carvacrol & p-cymene & 15.7 & 2.5 & 15.3 & 16.4 & 0.1 & No effect \\
\hline carvacrol & eugenol & 15.7 & 2.8 & 15.6 & 48.7 & 70.3 & Synergistic \\
\hline carvacrol & myrcene & 15.7 & 18.6 & 28.9 & 71.3 & 62.1 & Synergistic \\
\hline carvacrol & $\beta$-pinene & 15.7 & 2.7 & 15.5 & 36.6 & 28.7 & Synergistic \\
\hline carvacrol & thymol & 15.7 & 3.1 & 15.8 & 56.4 & 103.8 & Synergistic \\
\hline thymol & cinnamaldehyde & 3.1 & 8.3 & 11.0 & 36.1 & 56.8 & Synergistic \\
\hline thymol & p-cymene & 3.1 & 2.5 & 5.4 & 4.6 & 0.1 & No effect \\
\hline thymol & eugenol & 3.1 & 2.8 & 5.7 & 34.8 & 147.9 & Synergistic \\
\hline thymol & myrcene & 3.1 & 18.6 & 21.0 & 58.7 & 67.5 & Synergistic \\
\hline thymol & $\beta$-pinene & 3.1 & 2.7 & 5.6 & 3.5 & 0.8 & No effect \\
\hline
\end{tabular}




\section{Discussion}

Essential oils can be used as part of insecticides that can affect disease-related insect vectors, but with low impact on the accompanying insect fauna $[29,30]$. There are reports on essential oils with insecticidal and inhibitory oviposition capacity against A. aegypti [28]. The potential biological activity of the different essential oils varies according to plant species, its origin and its composition [31,32].

Regarding the larvicidal activities evaluated on instar III, there were no differences among the essential oils studied, except for the cinnamon essential oil (C. verum J. Presl), which has a variation on $\mathrm{LC}_{50}$ and $\mathrm{LC}_{90}$ values. Results included in Table 2 demonstrate that the essential oils tested can effectively control C. quinquefasciatus pupae and larvae, and the effect can be the result of the interaction of the many compounds found in each essential oil, many in small or trace quantities, or can be attributed mainly to major components. To answer the latter question, the larvicidal and pupaecidal activity of the chemical compounds reported as major constituents of the essential oils tested were analyzed, and the results are shown in Table 3.

Lethal concentration tests $\left(\mathrm{LC}_{50}\right.$ and $\left.\mathrm{LC}_{90}\right)$ provide information on the concentration of a given compound to decrease a population by $50 \%$ and $90 \%$, respectively. Usually the effect is not linear; therefore, it is important to determine both values. In the determination of those values, at least three replicates are needed for each concentration in the analysis, and this is the reason for the report of confidence intervals (provided in parenthesis in Tables 2 and 3). However, it is not possible to assess differences among the essential oil or the main components, so the analysis of larval mortality was also included in this work. The essential oils and their main components included in this study also showed a high effectiveness with respect to mortality upon exposure of Culex quinquefasciatus larvae to water contaminated with lethal doses (Table 4): in most cases, deaths occurred after short-term exposures.

Results of larval mortality demonstrated that the activity of anise essential oil and trans-anethole are statistically similar to the activity of Temephos, while L. nobilis L. essential oil and its main component (eucalyptol) were less effective. Cinnamaldehyde, the major component of cinnamon essential oil, showed higher $\mathrm{LC}_{50}$ compared to the essential oil, suggesting that there are antagonistic effects of some components present in cinnamon essential oil. On the contrary, cuminaldehyde the major constituent of cumin essential oil, is more active than the essential oil.

Eugenol and eucalyptol, by themselves had better activity than the essential oil where they are present. In some cases, such as with eucalyptol that is present in bay laurel essential oil, the larvicidal activity doubles when the molecule is pure. This suggests that the other constituents of the essential oil have antagonistic interactions with eucalyptol. The low larvicidal activity of eucalyptol (1,8-cineol) has been reported previously against larvae of Aedes aegypti [33], Anopheles anthropophagus [34] and Culex pipiens [35], however it has been shown to be effective as a repellent in foods and highly effective as an ovipositional repellent [33], however the effect did not last for more than $30 \mathrm{~min}$ [36].

Limonene has a larvicidal activity similar to the essential oil of Mexican lime against stage III larvae of $C$. quinquefasciatus. Its activity doubled when the molecule was evaluated as a pure compound on pupae. This suggests that other components of the essential oil have an antagonist effect with limonene or that the compound is more effective against larvae in a more mature stage. These results demonstrate the importance of studying the components of the essential oils separately, as different studies could rule out some important molecules because the oil as a whole has seemingly unimportant biological activities. Eleni et al. [37] reported the larvicidal activity of limonene rich essential oil from Citrus auranitium subsp. bergamia against Culex pipiens; the oil displays a $\mathrm{LC}_{50}$ of $58.73 \mathrm{mg} / \mathrm{L}$. On the other hand, Mentha longifolia essential oil, containing 20\% limonene, has been reported as larvicidal against Culex pipiens with a $\mathrm{LC}_{50}$ of $78.28 \mathrm{mg} / \mathrm{L}$ after $48 \mathrm{~h}$ of exposure [38].

There are multiple plant species collectively known as Oregano, including plants from the Verbenaceae and the Lamiaceae families, that have in common the presence of thymol and carvacrol in different proportions, but with similar odor and flavor characteristics [22] and, as such, are comparable in biological activities. In this study, the essential oil of Mexican oregano (Lippia berlandieri Schauer) has carvacrol as its major constituent (57\%), followed by thymol as the second major compound. 
There is no difference between the larvicidal activity of carvacrol and the essential oil of Mexican oregano, but the larvicidal activity of thymol is significantly lower. The only structural difference between carvacrol and thymol is the position of the hydroxyl group on the benzene ring with respect to the largest aliphatic chain (Figure 1); this demonstrate the importance of the aliphatic chain and its proximity to other functional groups.

There are numerous reports on the insecticidal activity of the essential oils from Origanum species; major components such as carvacrol, thymol, $\gamma$-terpinene and terpinen-4-ol are reported with fumigant and repellent activity rather than contact toxicity [39]. Cetin and Yanikoglu determined the insecticidal activity of essential oils from two species of Origanum (Origanum onites L. and Origanum minutiflorum) on the third and fourth instar larvae of Culex pipiens L.: the $\mathrm{LC}_{50}$ values were 24.8 and $73.8 \mu \mathrm{g} / \mathrm{mL}$, respectively [40]. The authors attribute the larvicidal activity to carvacrol, as also suggested in this report. However, it is important to note that other studies have reported that oregano essential oil with high levels of thymol have an effective deterrent oviposition activity against Culex quinquefasciatus females [41].

Regarding essential oils derived from plants of the genus Lippia, this study is the first to report the activity of the essential oil of Lippia berlandieri Shauer. Vera et al. evaluated the activity of Lippia alba and Lippia origanoides against larvae of Aedes aegypti [42]. These two essential oils contained carvone and carvacrol as major components, and presented $\mathrm{LC}_{50}$ values of approximately $50 \mu \mathrm{g} / \mathrm{mL}$. In addition, the essential oils of Lippia gracilis and Lippia sidoides have been evaluated against Aedes aegypti [43,44]. Gleiser and Zygadlo evaluated the activity of the essential oils of Lippia turbinata and Lippia polystachya against Culex quinquefasciatus larvae [45]. Both oils presented $\alpha$-thujone as the major component and showed no relevant larvicidal activity.

The larvicidal efficiency of anise essential oil as well trans-anethole, has been previously reported [46]. Waliwitiya et al., Pavela and Sousa et al. evaluated the activity of trans-anethole against larvae of Aedes aegypti, Anopheles atroparous and Culex quinquefasciatus respectively, finding relevant $\mathrm{LC}_{50}$ values [47-49]. This study confirms the activity of Pimpinella anisum L. essential oil, but it shows that trans-anethole does not eliminate the larvae as quickly as it does the essential oil, even though both have similar $\mathrm{LC}_{50}$ values. This suggests that there are interactions with other components that are present in the essential oil at lower or even trace concentrations. Therefore, it is always important to analyze the activity of the complete essential oil and their major components, since the presence of compounds different than the main components, can present synergistic or antagonistic effects.

The most relevant synergistic effects are observed against larvae with the combination of trans-anetol and limonene and the combination of trans-anethole and $\beta$-myrcene against pupae. These effects may be responsible for the rapid larvicidal activity of the essential oil of P. anisum. Synergistic effects are also observed when mixing carvacrol and thymol, the main constituents of Lippia berlandieri essential oil. However, none of these compounds has additive effects on the activity when they are mixed with $p$-cymene. The only antagonistic effect was observed with the limonene-pinene combination, which may be related to the difference in activity between C.aurantifolia essential oil and limonene against pupae.

The mechanism of action with which the essential oils exert their larvicidal activity is not completely described. Pratti et al. suggest that instant death is due to severe damage of Malpighian tubules, since they are responsible for the excretion, not only of electrolytes and metabolites, but also of the high volume of water naturally present in insect larvae environment [50]. There are reports suggesting that spice essential oils have neurotoxic poisoning-type effect on insects, similar to the ones produced by organophosphate and carbamate insecticides, by inhibition of the of the acetylcholinesterase enzyme (AChE) [51]. In a comparative study of the vapor action of essential oils from plants of the Lamiaceae family on R. dominica adults, it was observed that the essential oils inhibited about $65 \%$ of AChE activity, while limonene lowered only $2 \%$ of the enzymatic activity [52]. Furthermore, these authors found that the essential oils significantly increased the levels of cyclic 
adenosine monophosphate (AMP) (even at very low concentrations), which suggests a possible action on octopamine. Similar results were obtained by Enan in flies and cockroaches exposed to eugenol and $\alpha$-terpineol [53]. In silico studies have demonstrated that terpenes can interact with AChE of A. aegypti, by joining a hydrophobic site of the enzyme, with an interaction with glycine 412, 409, abd 412 and isoleucine 413 amino acids [54]. Another proposed mechanism of action has been presented by Priestley et al., who suggest that thymol acts on GABA receptors of Drosophila melanogaster [55].

\section{Conclusions}

In conclusion, the high larvicidal activity of essential oils and their constituent compounds make them potential substitutes for traditionally used chemical compounds in larval and pupal stages of mosquito control programs. The essential oil is readily available and the cost constraint can be overcome by the low value of the $\mathrm{LC}_{50}$. The larvicidal activity of Essential oils shows that it is not necessary to use the pure active compound, since the complex mixture of compounds present in essential oils are effective as larvicide.

On the other hand, their principal constituents are low molecular weight compounds and therefore easily synthesized and the study of the pure components is essential to elucidate the larvicidal and insecticidal mechanism of action of essential oils and their constituents.

Acknowledgments: Sergio Andrade-Ochoa and Karla Fabiola Chacón-Vargas would like to thank Consejo Nacional de Ciencia y Tecnología (CONACYT) for scholarship for their graduate studies. Sergio Andrade-Ochoa wishes to acknowledge the support of Intituto Chihuahuense de la Juventud (ICHIJUV) and Eric Contreras-Suarez in carrying out this work.

Author Contributions: Benjamin Nogueda-Torres and Luvia E. Sánchez-Torres supervised the larvicidal bioassay, conducted by Sergio Andrade-Ochoa and D.S.A. Sergio Andrade-Ochoa, Alejandro D. Camacho and Benjamin Nogueda-Torres evaluated the oviposition deterrent activities. Sergio Andrade-Ochoa, Karla Fabiola Chacón-Vargas and Blanca E. Rivera-Chavira conducted the kinetics bioassays. Sergio Andrade-Ochoa, Luvia E. Sánchez-Torres and Karla Fabiola Chacón-Vargas evaluated the synergism/antagonism effects. Alejandro D. Camacho and Guadalupe Virginia Nevárez-Moorillón carried out the statistical analysis. Guadalupe Virginia Nevárez-Moorillón and Sergio Andrade-Ochoa wrote the first draft of the manuscript. Guadalupe Virginia Nevárez-Moorillón conceived the study and participated in its design and coordination. All authors read and agreed with the final version of this manuscript.

Conflicts of Interest: The authors declare that they have no conflict of interest.

\section{References}

1. Estrada-Aguilar, A.; Sánchez-Manzano, R.M.; Martínez-Ibarra, J.A.; Camacho, A.D.; Márquez-Navarro, A.; Nogueda-Torres, B. Larvicidal activity of micronized aqueous suspension of calcium hydroxide against Aedes aegypti and Culex quinquefasciatus (Diptera: Culicidae). Parasitol. Res. 2012, 110, 1091-1095. [CrossRef] [PubMed]

2. Taylor, M.J.; Hoerauf, A.; Bockarie, M. Lymphatic filariasis and onchocerciasis. Lancet 2010, 376, 1175-1185. [CrossRef]

3. Raghavendra, K.; Barik, T.K.; Bhatt, R.M.; Srivastava, H.C.; Sreehari, U.; Dash, A.P. Evaluation of the pyrrole insecticide chlorfenapyr for the control of Culex quinquefasciatus Say. Acta Trop. 2011, 118, 50-55. [CrossRef] [PubMed]

4. Karunamoorthy, K.; Ilango, K.; Murugan, K. Laboratory evaluation of traditionally used plant-based insect repellents against the malaria vector Anopheles arabiensis Patton. Parasitol. Res. 2010, 106, 1217-1223. [CrossRef] [PubMed]

5. Ocampo, C.B.; Salazar-Terreros, M.J.; Mina, N.J.; McAllister, J.; Brogdon, W. Insecticide resistance status of Aedes aegypti in 10 localities in Colombia. Acta Trop. 2011, 118, 37-44. [CrossRef] [PubMed]

6. Miranda, J.E.; Navickiene, H.M.D.; Nogueira-Couto, R.H.; De Bartoli, S.A.; Kato, M.J.; Bolzani, V.S.; Furlan, M. Susceptibility of Apis mellifera (Hymenoptera: Apidae) to pellitorine, an amide isolated from Piper tuberculatum (Piperaceae). Apidologie 2003, 34, 409-415. [CrossRef] 
7. Lin, C.Y.; Wu, D.C.; Yu, J.Z.; Chen, B.H.; Wang, C.L.; Ko, W.H. Control of silver leaf whitefly, cotton aphid and kanzawa spider mite with oil and extracts from seeds of sugar apple. Neotrop. Entomol. 2009, 38, 531-536. [CrossRef] [PubMed]

8. McCarroll, L.; Hemingway, J. Can insecticide resistance status affect parasite transmission in mosquitoes? Insect Biochem. Mol. Biol. 2002, 32, 1345-1351. [CrossRef]

9. Enayati, A.A.; Hemingway, J. Pyrethroid insecticide resistance and treated bednets efficacy in malaria control. Pestic. Biochem. Physiol. 2016, 84, 116-126. [CrossRef]

10. Kumar, S.; Mishra, M.; Wahab, N.; Warikoo, R. Larvicidal, repellent, and irritant potential of the seed-derived essential oil of Apium graveolens against dengue vector, Aedes aegypti L. (Diptera: Culicidae). Front. Public Health. 2014, 2, 147. [CrossRef] [PubMed]

11. Chowdhury, N.; Ghosh, A.G. Chandra, Mosquito larvicidal activities of Solanum villosum berry extract against the dengue vector Stegomyia aegypti. BMC Complement. Altern. Med. 2008, 3, 8-10.

12. Mdoe, F.P.; Cheng, S.S.; Lyaruu, L.; Nkwengulila, G.; Chang, S.T.; Kweka, E.J. Larvicidal efficacy of Cryptomeria japonica leaf essential oils against Anopheles gambiae. Parasit. Vectors 2011, 7, 426. [CrossRef] [PubMed]

13. Burt, S. Essential oils: Their antibacterial properties and potential applications in foods-A review. Int. J. Food Microbiol. 2004, 94, 223-253. [CrossRef] [PubMed]

14. Celikel, N.; Kavas, G. Antimicrobial properties of some essential oils against some pathogenic microorganisms. Czech J. Food Sci. 2008, 26, 174-181. [CrossRef]

15. Gachkar, L.; Rasooli, I.; Yadegari, D.; Bagher-Rezaei, M.; Taghizadeh, M.; Alipoor-Astaneh, S. Chemical and biological characteristics of Cuminum cyminum and Rosmarinus officinalis essential oils. Food Chem. 2007, 102, 898-904. [CrossRef]

16. Pitasawat, B.; Champakaew, D.; Choochote, W.; Jitpakdi, A.; Chaithong, U.; Kanjanapothi, D.; Rattanachanpichai, E.; Tippawangkosol, P.; Riyong, D.; Tuetun, B.; et al. Aromatic plant-derived essential oil: An alternative larvicide for mosquito control. Fitoterapia 2007, 78, 205-210. [CrossRef] [PubMed]

17. Wafa, G.; Amadou, D.; Larbi, K.M. Larvicidal activity, phytochemical composition, and antioxidant properties of different parts of five populations of Ricinus communis L. Ind. Crops Prod. 2014, 56, 43-51. [CrossRef]

18. Panella, N.A.; Dolan, M.C.; Karchesy, J.J.; Xiong, Y.; Peralta-Cruz, J.; Khasawneh, M.; Montenieri, J.A.; Maupin, G.O. Use of novel compounds for pest control: Insecticidal and acaricidal activity of essential oils components from heartwood of Alaska yellow cedar. J. Med. Entomol. 2005, 42, 352-358. [CrossRef] [PubMed]

19. Pavela, R. Acute toxicity and synergistic and antagonistic effects of the aromatic compounds of some essential oils against Culex quinquefasciatus Say larvae. Parasitol. Res. 2015, 114, 3835-3853. [CrossRef] [PubMed]

20. Harwood, R.F.; James, M.T. Entomología Médica y Veterinaria, Primera Edición ed; LIMUSA: Ciudad de México, México, 1987; pp. 201-203.

21. Andrade-Ochoa, S.; Chacón-Vargas, K.F.; Nevárez-Moorillón, G.V.; Rivera-Chavira, B.E.; Hernández-Ochoa, L.R. Evaluation of antimycobacterium activity of the essential oils of cumin (Cuminum cyminum), clove (Eugenia caryophyllata), cinnamon (Cinnamomum verum), laurel (Laurus nobilis) and anis (Pimpinella anisum) against Mycobacterium tuberculosis. Adv. Biol. Chem. 2013, 3, 480-484.

22. Avila-Sosa, R.; Hernández-Zamoran, E.; López-Mendoza, I.; Palou, E.; Munguía, M.T.j.; Nevárez-Moorillón, G.V.; López-Malo, A. Fungal inactivation by Mexican oregano (Lippia berlandieri Schauer) essential oil added to amaranth, chitosan, or starch edible films. J. Food Sci. 2010, 75, M127-M133. [CrossRef] [PubMed]

23. Sanchez-Aldana, D.S.; Andrade-Ochoa, S.; Aguilar, C.N.; Contreras-Esquivel, J.C.; Nevárez-Moorillón, G.V. Antibacterial activity of pectic-based edible films incorporated with Mexican Lime essential oil. Food Control 2014, 50, 907-912. [CrossRef]

24. Pavela, R.; Kaffkova, K.; Kumsta, M. Chemical composition and larvicidal activity of essential oils from different Mentha L. and Pulegium species against Culex quinquefasciatus Say (Diptera: Culicidae). Plant Protect. Sci. 2004, 50, 36-42. [CrossRef]

25. Xue, R.D.; Barnard, D.R.; Ali, A. Laboratory and field evaluation of insect repellents as oviposition deterrents against the mosquito Aedes albopictus. Med. Vet. Entomol. 2011, 15, 126-131. [CrossRef]

26. Ajaiyeoba, E.O.; Sama, W.; Essien, E.E.; Olayemi, J.O.; Ekundayo, O.; Walker, T.M.; Setzer, W.N. Larvicidal Activity of Turmerone-Rich Essential Oils of Curcuma longa. Leaf and Rhizome from Nigeria on Anopheles gambiae. Pharm. Biol. 2008, 46, 279-282. 
27. Hummelbrunner, L.A.; Isman, M.B. Acute, sublethal, antifeedant, and synergistic effects of monoterpenoid essential oil compounds on the tobacco cutworm, Spodoptera litura (Lep., Noctuidae). J. Agric. Food Chem. 2001, 49, 715-720. [CrossRef] [PubMed]

28. Coria, C.; Almiron, W.; Valladares, G.; Carpinella, C.; Ludueña, F.; Defago, M.; Palacios, S. Larvicidae and oviposition deterrent effects of fruit and leaf ext from Melia azedarach (L) on Aedes aegypti (L) (Diptera: Culicidae). Bioresour. Technol. 2008, 99, 3066-3070. [CrossRef] [PubMed]

29. Badreddine, B.S.; Olfa, E.; Samir, D.; Hnia, C.; Lahbib, B.J.M. Chemical composition of Rosmarinus and Lavandula essential oils and their insecticidal effects on Orgyia trigotephras (Lepidoptera, Lymantriidae). Asian Pac. J. Trop. Med. 2015, 8, 98-103. [CrossRef]

30. Isman, M. Plant essential oil for pest and disease management. Crop Prot. 2000, 19, 603-608. [CrossRef]

31. Carvalho, A.F.U.; Melo, V.M.M.; Craveiro, A.A.; Machado, M.I.L.; Bantim, M.B.; Rabelo, E.F. Larvicidal activity of the essential oil from Lippia sidoides Cham. against Aedes aegypti Linn. Mem. Inst. Oswaldo Cruz. 2003, 98, 569-571. [CrossRef] [PubMed]

32. Lee, H.S. Mosquito larvicidal activity of aromatic medicinal plants oils against Aedes aegypti and Culex pipiens pallens. J. Am. Mosq. Control Assoc. 2006, 22, 292-295. [CrossRef]

33. Klocke, J.A.; Darlington, M.V.; Balandrin, M.F. 1,8-Cineole (Eucalyptol), a mosquito feeding and ovipositional repellent from volatile oil of Hemizonia fitchii (Asteraceae). J. Chem. Ecol. 1987, 13, 2131-2141. [CrossRef] [PubMed]

34. Cheng, S.S.; Huang, C.G.; Chen, Y.J.; Yu, J.J.; Chen, W.J.; Chang, S.T. Chemical compositions and larvicidal activities of leaf essential oils from two eucalyptus species. Bioresour. Technol. 2009, 100, 452-456. [CrossRef] [PubMed]

35. Perumalsamy, H.; Kim, N.J.; Ahn, A.J. Larvicidal activity of compounds isolated from Asarum heterotropoides against Culex pipiens pallens, Aedesa egypti, and Ochlerotatus togoi (Diptera: Culicidae). J. Med. Entomol. 2009, 46, 1420-1423. [CrossRef] [PubMed]

36. Alvarez Costa, A.; Naspi, C.V.; Lucia, A.; Masuh, H.M. Repellent and Larvicidal Activity of the Essential Oil from Eucalyptus nitens Against Aedes aegypti and Aedes albopictus (Diptera: Culicidae). J. Med. Entomol. 2017, 54, 670-676. [PubMed]

37. Eleni, M.; Antonies, M.; George, K.; Alexios-Leandos, S.; Prokopios, M. High quality bergamot oil from Greece: Chemical analysis using chiral gas chromatography and larvicidal activity against the West Nile virus vector. Molecules 2009, 14, 839-849. [CrossRef] [PubMed]

38. Koliopoulos, G.; Pitarokili, D.; Kioulos, E.; Michaelakis, A.; Tzako, O. Chemical composition and larvicidal evaluation of Mentha, Salvia and Melissa essential oils against the West Nile virus mosquito Culex pipiens. Parasitol. Res. 2010, 107, 327-333. [CrossRef] [PubMed]

39. Erler, F. Fumigant activity of six monoterpenoids from aromatic plants in Turkey against the two stored-product insects confused flour beetle, Tribolium confusum, and Mediterranean flour moth, Ephestia kuehniella. J. Plant Dis. Prot. 2005, 112, 602-611. [CrossRef]

40. Cetin, H.; Yanikoglu, A. A study of the larvicidal activity of Origanum (Labiatae) species from southwest Turkey. J. Vector Ecol. 2006, 31, 118-122. [CrossRef]

41. Pavela, R. Larvicidal property of essential oils against Culex quinquefasciatus Say (Diptera: Culicidae). Ind. Crops Prod. 2009, 30, 311-315. [CrossRef]

42. Vera, S.S.; Zambrano, D.F.; Méndez-Sanchez, S.C.; Rodríguez-Sanabria, F.; Stashenko, E.E.; Luna, J.E.D. Essential oils with insecticidal activity against larvae of Aedes aegypti (Diptera: Culicidae). Parasitol. Res. 2014, 113, 2647-2654. [CrossRef] [PubMed]

43. Silva, W.J.; Dória, G.A.A.; Maia, R.T.; Nunes, R.S.; Carvalho, G.A.; Blank, A.F.; Alves, P.B.; Marçal, R.M.; Cavalcanti, S.C.H. Effects of essential oils on Aedes aegypti larvae: Alternatives to environmentally safe insecticides. Bioresour. Technol. 2008, 99, 3251-3255. [CrossRef] [PubMed]

44. De Lima, G.P.G.; de Souza, T.M.; de Paula Freire, G.; Farias, D.F.; Cunha, A.P.; Ricardo, N.M.P.S.; de Morais, S.M.; Carvalho, A.F.U. Further insecticidal activities of essential oils from Lippia sidoides and Croton species against Aedes aegypti L. Parasitol. Res. 2013, 112, 1953-1958. [CrossRef] [PubMed]

45. Gleiser, R.M.; Zygadlo, J.A. Insecticidal properties of essential oils from Lippia turbinata and Lippia polystachya (Verbenaceae) against Culex quinquefasciatus (Diptera: Culicidae). Parasitol. Res. 2007, 101, 1349-1354. [CrossRef] [PubMed] 
46. Prajapati, V.; Tripathi, A.K.; Aggarwal, K.K.; Khanuja, S.P.S. Insecticidal, repellent and oviposition-deterrent activity of selected essential oils against Anopheles stephensi, Aedes aegypti and Culex quinquefasciatus. Bioresour. Technol. 2005, 96, 1749-1757. [CrossRef] [PubMed]

47. Waliwitiya, R.; Kennedy, C.J.; Lowenberger, C.A. Larvicidal and oviposition-altering activity of monoterpenoids, trans-anethole and rosemary oil to the yellow fever mosquito Aedes aegypti (Diptera: Culicidae). Pest Manag. Sci. 2009, 65, 241-248. [CrossRef] [PubMed]

48. Pavela, R. Insecticidal properties of Pimpinella anisum essential oils against the Culex quinquefasciatus and the non-target organism Daphnia magna. J. Asia Pac. Entomol. 2014, 17, 287-293. [CrossRef]

49. Sousa, R.M.O.; Rosa, J.S.; Silva, C.A.; Almeida, M.T.M.; Novo, M.T.; Cunha, A.C.; Fernandes-Ferreira, M. Larvicidal, molluscicidal and nematicidal activities of essential oils and compounds from Foeniculum vulgare. J. Pest Sci. 2015, 88, 413-426. [CrossRef]

50. Pratti, D.L.; Ramos, A.C.; Scherer, R.; Cruz, Z.M.; Silva, A.G. Mechanistic basis for morphological damage induced by essential oil from Brazilian pepper tree, Schinus terebinthifolia, on larvae of Stegomyia aegypti, the dengue vector. Parasit. Vectors 2015, 8, 136. [CrossRef] [PubMed]

51. Houghton, P.J.; Ren, Y.; Howes, M.J. Acetylcholinesterase inhibitors from plants and fungi. Nat. Prod. Rep. 2006, 23, 181-199. [CrossRef] [PubMed]

52. Kostyukovsky, M.; Rafaeli, A.; Gileadi, C.; Demchenko, N.; Shaaya, E. Activation of octopaminergic receptors by essential oil constituents isolated from aromatic plants: Possible mode of action against insect pests. Pest Manag. Sci. 2002, 58, 1101-1106. [CrossRef] [PubMed]

53. Enan, E. Insecticidal activity of essential oils: Octopaminergic sites of action. Comp. Biochem. Physiol. C Toxicol. Phar. 2001, 130, 325-337. [CrossRef]

54. Praveena, A.; Sanjayan, K.P. Inhibition of acetylcholinesterase in three insects of economic importance by linalool, a monoterpene phytochemical. In Insect Pest Management, a Current Scenario, Entomology Research Unit; Ambrose, D.P., Ed.; St. Xavier's College: Palayamkottai, India, 2011; pp. 340-345.

55. Priestley, C.M.; Williamson, E.M.; Wafford, K.A.; Satelle, D.B. Thymol, a constituent of thyme essential oil, is a positive allosteric modulator of human GABAA receptors and a homo-oligomeric GABA receptor from Drosophila melanogaster. Br. J. Pharmacol. 2003, 140, 1363-1372. [CrossRef] [PubMed]

(C) 2018 by the authors. Licensee MDPI, Basel, Switzerland. This article is an open access article distributed under the terms and conditions of the Creative Commons Attribution (CC BY) license (http:/ / creativecommons.org/licenses/by/4.0/). 